\title{
Variación semántica y significado social: hacia una sociolingüística cognitiva de la Tercera Ola
}

\author{
Andrea PIZARRO PEDRAZA \\ Université catholique de Louvain \\ andrea.pizarro@uclouvain.be
}

\begin{abstract}
RESUMEN
Dos elementos clave revelan un cambio de etapa en sociolingüística hacia una perspectiva más flexible: el estudio de la variación semántica y la inclusión de variables microsociológicas. Este artículo consiste en una propuesta teórica para combinar las dos perspectivas responsables de este cambio, respectivamente, la sociolingüística cognitiva y la Tercera Ola de los estudios de variación. Se localizan las conexiones teóricas entre ambas y se expone el programa para una sociolingüística cognitiva de la Tercera Ola. Combinando objetivos, métodos y herramientas de ambas, se subraya la necesidad de investigar la capacidad de la variación semántica para crear significado social. A diferencia de los trabajos sociolingüístico-cognitivos hasta ahora, se defiende una perspectiva 'más sociolingüística' tanto en la obtención de datos, como en la interpretación del significado de la variación para los hablantes en tanto que agentes sociales, como en la Tercera Ola de los estudios de variación.
\end{abstract}

Palabras clave: sociolingüística cognitiva, Tercera Ola, variación semántica.

\begin{abstract}
Two key elements reveal a step change in Sociolinguistics towards a more flexible perspective: the study of semantic variation and the inclusion of microsociological variables. This article is a theoretical proposal to combine the two perspectives that are responsible for this change: respectively, Cognitive Sociolinguistics and the Third Wave of Variation Studies. The theoretical connections between the two are located and the program for a Third Wave Cognitive Sociolinguistics is exposed. Combining objectives, methods and tools, this paper emphasizes the need to investigate how semantic variation creates social meaning. Unlike cognitive sociolinguistic works so far, the proposal defendes a "more sociolinguistic" perspective, both in data collection and in the interpretation of the meaning of variation for speakers as social agents, as in the Third Wave.
\end{abstract}

Key words: Cognitive Sociolinguistics, Third Wave, Semantic variation. 


\section{Introducción}

Ciertas propuestas recientes para el estudio de la variación sociolingüística han abierto caminos para la investigación de fenómenos relevantes y no suficientemente explorados. Las páginas siguientes abordarán dos de ellos: la variación semántica y las variables microsociológicas construidas en el discurso. Aunque puedan parecer elementos dispares, ambos encarnan una etapa menos rígida de la sociolingüística y, como tal, serán las claves de la propuesta teórica y metodológica de este artículo.

Esta argumentación tratará de complementar a la sociolingüística cognitiva ${ }^{1}$, que ha abierto la puerta al estudio variacionista de la semántica léxica ${ }^{2}$. La sociolingüística cognitiva reclama la introducción de factores sociales, además de los propiamente lingüísticos, en los trabajos cognitivistas de variación, para abordar empíricamente una de las bases teóricas de la lingüística cognitiva: la base social y cultural de la cognición ${ }^{3}$.

Aunque la sociolingüística cognitiva es un esfuerzo interdisciplinar, hasta ahora las contribuciones han sido mayoritariamente cognitivistas ${ }^{4}$, inclinando la balanza metodológica y, consecuentemente, interpretativa, hacia aspectos afines a ese origen. Entre otros, la sociolingüística cognitiva no incorpora sistemáticamente postulados sociolingüísticos de la Tercera $\mathrm{Ola}^{5}$, que han supuesto un giro en la interpretación del significado de la variación, vinculado precisamente con el concepto de variables microsociológicas en detrimento de las macrosociológicas tradicionales, consideradas demasiado categóricas. Si bien Geeraerts y Kristiansen ${ }^{6}$ reconocen explícitamente el interés potencial de la combinación con la Tercera Ola, las líneas de la sociolingǘstica cognitiva acordes con los desarrollos sociolingüísticos recientes son minoritarias ${ }^{7}$ y la reflexión teórica debe ser plenamente abordada. Este artículo pretende contribuir a la disciplina desde la sociolingüística, como reclaman algunos autores ${ }^{8}$, incorporando elementos tradicionales y otros recientes.

A continuación, haré algunas propuestas sobre cómo podría ser ese encuentro entre la sociolingüística cognitiva y la Tercera Ola. Para ello, este artículo tratará de

\footnotetext{
${ }^{1}$ D. Geeraerts y G. Kristiansen (2014); G. Kristiansen y R. Dirven (2008); D. Geeraerts, G. Kristiansen, e Y. Peirsman (2010).

${ }^{2} \mathrm{Me}$ interesa esta línea en concreto, más que los planteamientos de Labov sobre el mecanismo cognitivo ligado a la percepción de las variables sociolingüísticas, especialmente las gramaticales y fonológicas, bautizado como "sociolinguistic monitor" (Labov et al. 2011), no directamente ligados al desarrollo del estudio de la variación semántica.

${ }^{3}$ G. Kristiansen y R. Dirven (2008), p. 3; D. Geeraerts (2005), p. 168-182.

${ }^{4}$ W. B. Hollmann (2013), p. 507.

${ }^{5}$ P. Eckert $(2005,2009)$.

${ }^{6}$ D. Geeraerts y G. Kristiansen (2014).

${ }^{7}$ G. Kristiansen (2006); E. Zenner, D. Speelman, y D. Geeraerts (2014).

${ }^{8}$ M. Pütz, J. A. Robinson, y M. Reif (2012); W. B. Hollmann (2013).
} 
contestar a la pregunta de investigación: ¿Qué puede ofrecer la Tercera Ola a la sociolingüística cognitiva? Esta pregunta es una vuelta de tuerca a las planteadas por Geeraerts y Kristiansen', y guiará un razonamiento 'más sociolingüístico' y, espero, complementario.

Reconociendo de antemano la necesaria simplificación, este artículo tratará los aspectos siguientes: primero, repasaré el problema semántico en sociolingüística. Posteriormente, presentaré la propuesta integradora de la sociolingüística $\operatorname{cognitiva}^{10}$, con hincapié en la semántica Léxica, y algunos de sus estudios. En contraste, resumiré los fundamentos de la Tercera Ola en cuanto a la interpretación del significado de la variación. Finalmente, analizaré la pertinencia de proponer un programa teórico, metodológico y analítico para una sociolingüística cognitiva de la Tercera Ola.

\section{El problema de la variable semántica en sociolingüística}

Antes de empezar con mi exposición, quisiera aclarar el uso que haré de algunos términos. Este artículo se centra principalmente en el nivel de la "variación semántica", es decir, la variación en el nivel de la semántica léxica, aquel que se ocupa de las relaciones entre las palabras y sus significados. La variación semántica se estudia en dos niveles: el semasiológico, que parte de la palabra y estudia la manifestación de sus diversos significados; y el onomasiológico, (formal o conceptual), que parte del concepto y se centra en las diversas palabras o expresiones que lo nombran ${ }^{11}$. En la variación onomasiológica puramente formal, no se implican distinciones de significado entre las dos o más palabras referidas al mismo concepto, como por ejemplo, en las variantes geográficas de tipo acálaquí y allíallá. Por otro lado, la variación onomasiológica conceptual es la que se da cuando las distintas formas de nombrar un concepto son categorías conceptuales distintas, como el uso de cáncer/enfermedad (hipónimo/hiperónimo) para referirse al mismo concepto 'cáncer'. Estos usos son muy habituales en el uso. Me interesa puntualizar que la denominada "variación léxica", común en sociolingüística, estudia la variación desde el punto de vista de las palabras, más que de la variación en los significados, por lo que se sitúa más bien en el plano onomasiológico formal. Quisiera precisar también que, al hablar de significado en estos casos, me refiero al

${ }^{9}$ D. Geeraerts y G. Kristiansen (2014).

${ }^{10}$ Los lectores interesados en ampliar nociones que, por cuestiones de espacio, no pueden ser más que esbozadas en este artículo, pueden dirigirse a algunas publicaciones en español muy adecuadas para ese fin, tanto en el ámbito de la lingüística cognitiva, como IbarretxeAntuñano y Valenzuela (2012a); Ibarretxe-Antuñano y Valenzuela (2012b); Cuenca y Hilferty (2013), como de la sociolingüística cognitiva, en Moreno Fernández (2012). No existen, hasta donde sé, publicaciones que sinteticen la Tercera Ola en español, por lo que referiré en su momento a los trabajos en inglés.

${ }^{11}$ D. Geeraerts, S. Grondelaers, y P. Bakema (1994). 
significado lingüístico. Más adelante, utilizaré otro concepto distinto, el significado social, que se refiere a las asociaciones de las variantes lingüísticas con aspectos extralingüísticos de la identidad social del hablante.

Para entender el impacto de la sociolingüística cognitiva, es necesario recordar el vacío semántico de la sociolingüística. Aunque se admite la variabilidad en todos los niveles de lengua y se reclama que todo estudio lingüístico debe ser sociolingüístico $^{12}$, la variación semántica ha sido desatendida en todos los niveles, especialmente en el onomasiológico conceptual, a excepción de los estudios de variación léxica (generalmente en perspectiva diatópica) y de los de léxico disponible, que mencionaré muy brevemente más adelante ${ }^{13}$. Los estudios variacionistas la consideran extremadamente problemática debido a la metodología original para la selección de las variables, a la que subyace una visión de la lengua que separa langue y parole. $^{14}$

Para la sociolingüística variacionista laboviana, una variable es una unidad del sistema lingüístico con variantes sensibles al contexto (extra)lingüístico ${ }^{15}$. Las variantes deben tener el mismo valor referencial o función gramatical ${ }^{16}$. Las variables deben ser muy frecuentes, estar insertas en la estructura gramatical y presentar estratificación social o estilística en su distribución ${ }^{17}$. Dada la aspiración de estudiar el vernáculo, las variables deben realizarse inconscientemente.

Estos requisitos resultan problemáticos para todos los niveles lingüísticos exceptuando el fonológico ${ }^{18} \mathrm{y}$, en menor medida, el morfológico ${ }^{19}$; pero el nivel sintáctico se enfrenta a la falta de equivalencia, a la dificultad en la identificación del contexto de la variable, y a la escasez de ocurrencias ${ }^{20}$. Estos problemas se reproducen en el plano léxico y, aún en mayor medida, en el semántico. Primeramente, la equivalencia de variantes choca con el problema de la sinonimia. Según López Morales ${ }^{21}$, dos palabras son variantes si tienen los mismos constituyentes semánticos, el mismo valor de verdad y son intercambiables en todos los contextos ${ }^{22}$. Este criterio ha acotado el estudio a alternancias léxicas en las que la equivalencia (total o parcial) plantea menos problemas, como

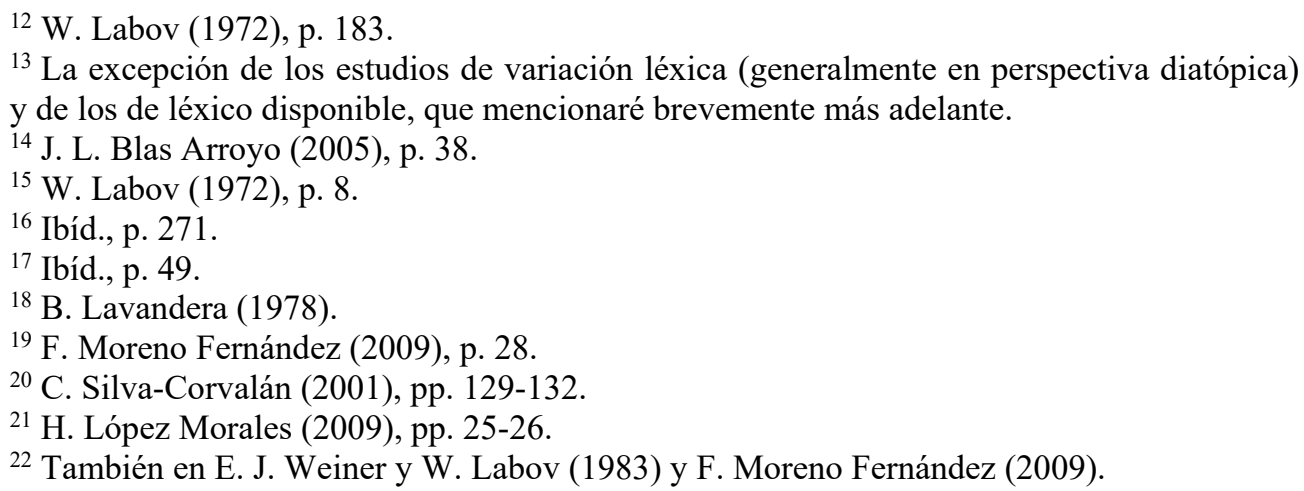


mujer/esposa/señora ${ }^{23}$, conocido, famoso y célebre; casa, domicilio, vivienda y residencia; fácil, simple y sencillo, etc. ${ }^{24}$.

Los demás criterios variacionistas también plantean problemas: tanto palabras concretas como conceptos tienen frecuencias muy bajas, incluso los muy comunes, y son realizados con un alto grado de consciencia. También se dan dificultades metodológicas para la cuantificación, ya que el análisis variacionista requiere listar todas las variantes posibles de la variable ${ }^{25}$, para conocer su peso específico. Esto es prácticamente imposible, dado el carácter abierto de los repertorios léxico y semántico ${ }^{26}$; especialmente en el nivel onomasiológico conceptual, ya que, en la negociación interactiva del significado, se pueden tratar como equivalentes elementos teóricamente alejados ${ }^{27}$, imposibilitando la delimitación a priori de variantes.

De estas limitaciones metodológicas se derivan además dos problemas teóricos para el estudio semántico: la concepción del sistema de la lengua y la ausencia de una teoría del significado lingüístico. Aunque los sociolingüistas variacionistas defendieran que existe un solo sistema de lengua y uso ${ }^{28}$, en la práctica, la necesidad de que exista un nivel de elementos subyacentes y otro en el que se realizan sus variantes hace muy difícil creer en un sistema lingüístico unitario, porque se siguen considerando dos planos, uno variable, y otro invariable y definible ${ }^{29}$. Paralelamente, la metodología sociolingüística se basa en la ausencia de diferencias semánticas, como ya se ha mencionado, priorizando variables como las fonológicas; por lo que no se desarrolló una teoría del significado lingüístico propiamente dicho ${ }^{30}$.

Estas dificultades han sido destacadas también en varias ocasiones en el mundo hispánico, especialmente con referencia a la variación léxica ${ }^{31}$. Entre las líneas de investigación principales en el mundo hispánico, destaca el estudio del léxico disponible, que se ha llevado a cabo de forma coordinada en varias comunidades hispanohablantes, como el Proyecto Panhispánico, iniciado por López Morales ${ }^{32}$. Estos estudios se centran principalmente en el plano onomasiológico formal, más

${ }^{23}$ F. Rodríguez González y B. L. Rochet (1999).

${ }^{24}$ L. Escoriza Morera (2012), p. 268.

${ }^{25}$ W. Labov (1972b).

${ }^{26}$ J. L. Blas Arroyo (2005), p. 79.

${ }^{27}$ F. Moreno Fernández (2012), p. 106.

${ }^{28}$ U. Weinreich, W. Labov y M. Herzog (1968).

${ }^{29}$ Ver, a este respecto, las críticas de M. A. K. Halliday (1996), p. 412; o R. Hasan (2009).

${ }^{30}$ B. Lavandera (1978).

${ }^{31}$ J. Borrego Nieto (1994); H. López Morales (2006); L. Escoriza Morera (2012).

${ }^{32}$ Las contribuciones, muy numerosas, se pueden consultar en la página web del proyecto: www.dispolex.com (fecha de consulta: 10/05/2016). Sirvan como ejemplo las siguientes: J. A. Samper Padilla (2006); N. Hernández Muñoz (2006); M. Etxebarria Arostegui (1996). 
que conceptual (de hecho se suele hablar de "vocabulario", "voces", "lexías" y de "campos léxicos"), y utilizan métodos de recogida de datos habitualmente basados en cuestionarios escritos. En otros estudios de variación léxica, se proponen una serie de opciones entre las que el informante debe elegir, según ciertos condicionantes contextuales, como las alternancias estudiadas por Escoriza Morera, mencionadas anteriormente.

Por su parte, la variación más allá de los lexías, más allá de la necesidad de equivalencias en el nivel del sistema, la que puede entrañar diferencias de significado lingüístico entre variantes, como en el nivel de la onomasiología conceptual, se ha desatendido casi por completo en sociolingüística, y el desarrollo de metodologías para su estudio en uso está muy poco avanzado, a pesar del interés que comportan ${ }^{33}$. Aunque ha habido intentos de análisis semántico componencial ${ }^{34}$, las variables semánticas no se pueden dividir en atributos "suficientes y necesarios", en el plano del sistema, como un fonema. Incluso conceptos aparentemente sencillos presentan problemas importantes en este sentido, como ha demostrado empíricamente la semántica cognitiva ${ }^{35}$. Sin duda, este bloqueo teórico y práctico choca con la percepción de hablantes y lingüistas, que aprecian la capacidad de palabras y conceptos para construir significado social en el discurso; como Sapir ${ }^{36}$, que mencionaba el apodo como elemento de vinculación intragrupal, o Silverstein ${ }^{37}$, que destaca la función de shibboleths de ciertas palabras. Sin menospreciar las dificultades mencionadas, la sociolingüística debería ser capaz de estudiar este nivel, con el fin de observar las diferencias sociales, identitarias y culturales en la forma de conceptualizar la realidad ${ }^{38}$.

\section{La sociolingüística cognitiva y la variación semántica}

Los problemas de la sociolingüística con la semántica léxica mencionados hasta ahora han motivado, en parte, el desarrollo de la sociolingüistica cognitiva, una línea de investigación reciente nacida de la aproximación de la lingüística cognitiva (con la que comparte aparato teórico) a las metodologías de estudio de la lengua en contexto social, propias de la sociolingüística. Desde sus primeras manifestaciones, la sociolingüística cognitiva ha prestado una atención especial al problema del significado lingüístico en perspectiva sociolingüística y a las posibles formas de estudiarlo. Se parte de la premisa fundamental de que, debido a las características

\footnotetext{
${ }^{33}$ M. A. Aijón Oliva y M. J. Serrano (2010).

${ }^{34}$ D. Sankoff, P. Thibault, y H. Bérubé (1978).

35 J. Allwood 2009; y ver la introducción a H. Cuyckens, R. Dirven, y J.R. Taylor (2009).

${ }^{36}$ E. Sapir (1949), p. 16.

${ }^{37}$ M. Silverstein $(1992 ; 2003)$.

${ }^{38}$ F. Sharifian (2011).
} 
propias del significado, se necesita un marco teórico flexible y no esencialista acorde con sus complejidades ${ }^{39}$.

\subsection{La teoría cognitiva del significado}

La motivación fundamental de la sociolingüística cognitiva como línea de investigación mixta es que las dos líneas que combina, la lingüística cognitiva y sociolingüística, son complementarias ${ }^{40}$. Notablemente, la lingüística cognitiva puede aportar a la sociolingüística una teoría del significado lingüístico, cuya idea fundacional es que el lenguaje es un instrumento para organizar, procesar y transmitir información ${ }^{41}$. En este modelo, el significado es un proceso de categorización, es decir, de traducción del mundo, a través del lenguaje, en símbolos (las categorías) con los que representamos la realidad en nuestro pensamiento ${ }^{42}$. El significado presenta cuatro propiedades esenciales. La primera de ellas es que el significado parte siempre de una perspectiva determinada. En este sentido, no refleja la realidad de forma objetiva, sino que la interpreta, lo que implica a su vez que lo que se comunica siempre se estructura desde una visión particular. Así, las diferencias entre las construcciones activas o pasivas, los deícticos, las expresiones espaciales, temporales y, en general, todos los elementos linguüísticos contribuyen a una estructuración del significado desde cierto punto de vista $^{43}$. En segundo lugar, el significado es dinámico y flexible. Las categorías semánticas son una interpretación de la realidad $\mathrm{y}$, puesto que esta es cambiante, el significado no puede entenderse como una estructura rígida, sino adaptable a los cambios. La relación entre los significados se asemeja a los parecidos de familia, por lo que es gradual: los elementos de una determinada categoría serán más o menos típicos según las características que compartan con ella ${ }^{44}$. Un ejemplo clásico es el de la categoría de 'ave', en la que los 'gorriones', las 'águilas' o los 'pingüinos' son ejemplares con características comunes y otras distintas, que los sitúan en posiciones más centrales o más periféricas de la categoría. En tercer lugar, el significado es enciclopédico y no autónomo con respecto a otros conocimientos que se tienen del mundo, como lo que aprehendemos a través de nuestro cuerpo, pero también lo que nos llega por estar insertos en culturas y sociedades específicas. Esto se deriva de que el lenguaje no está separado del resto de las capacidades cognitivas. El aparato cognitivo en general no se compone de conceptos aislados, sino de bloques más amplios de significado a modo de escenas ${ }^{45}$. Así, la

\footnotetext{
${ }^{39}$ K. Janicki (2006).

${ }^{40}$ D. Geeraerts y G. Kristiansen (2014).

${ }^{41}$ D. Geeraerts (2006).

42 J. R. Taylor (1989).

${ }^{43}$ D. Geeraerts (2006), p. 4.

${ }^{44}$ Ibíd. p. 4.

45 J. Fillmore (1982).
} 
estructuración del significado depende de todas las experiencias que tenemos como personas, tanto desde el punto de vista sensorial como identitario ${ }^{46}$. Ligada a la anterior, la última característica del significado lingüístico es que está basado en el uso y en la experiencia, por lo que la mejor manera de observarlo es a través de ejemplos reales, y no creados ad hoc. Desde este punto de vista, la preeminencia tradicional del nivel estructural de la lengua frente al nivel del uso se rechaza; lógicamente, si se acepta que el conocimiento de la lengua es experiencial, la única forma de llegar a conocer su funcionamiento es a través del uso.

La sociolingüística cognitiva entiende la lengua como fragmentos de uso real que emergen por encima de las situaciones individuales como un 'potencial estructurado ${ }^{47}$, que sería el sistema. Geeraerts ${ }^{48}$ propone pensar en la lengua como un conjunto de sistemas lectales con rasgos de prototipicidad, cuyos fenómenos lingüísticos son más o menos centrales (o prototípicos) dentro de la variedad lectal. En esta visión dialéctica entre sistema y uso, el estudio de la lengua en contexto es la única forma de acceder a las regularidades del sistema ${ }^{49}$.

La sociolingüística cognitiva propone adoptar la metodología sociolingüística para estudiar sistemáticamente las variables contextuales y sociales. Así se conjugan las dos facetas de la experiencia que influyen sobre el significado, la experiencia corporeizada y la social; para probar si, como postulan, grupos distintos en diversas condiciones sociales, geográficas, etc., tienen un conocimiento del mundo distinto que conlleva diferencias en la conceptualización ${ }^{50}$.

En definitiva, el beneficio es mutuo. La lingüística cognitiva puede aprender de la sociolingüística en el análisis con variables sociales; y la sociolingüística puede incorporar una teoría del significado y una metodología para analizar la semántica léxica. Además, al incluir en la naturaleza social del ser humano un aparato cognitivo entre el mundo y la categorización, la comprensión del fenómeno de la variación es integral, ya que contempla el círculo contexto-cognición ${ }^{51}$.

\subsection{La aplicación práctica de la sociolingüística cognitiva}

La visión de la sociolingǘstica cognitiva tiene además consecuencias metodológicas. En la práctica, supone abrir el campo de estudio a variables con diferencias de significado. Más que un significado esencial, en el sentido platónico (cf. Cratilo), se considera que las categorías siguen los principios de

\footnotetext{
${ }^{46}$ D. Geeraerts (2006), p. 5.

${ }^{47}$ K. Boye y P. Harder (2007), p. 572.

${ }^{48}$ D. Geeraerts (2010).

${ }^{49}$ D. Geeraerts y G. Kristiansen (2014).

${ }^{50}$ G. Kristiansen y R. Dirven (2008), p. 4.

${ }^{51}$ F. Moreno Fernández (2012), pp. 18-19.
} 
prototipicidad $^{52}$. No pueden definirse según rasgos suficientes y necesarios, ya que no son discretas, ni homogéneas. Se rechaza así la tendencia sociolingüística a definir los conceptos sin ambigüedad y se opta por una visión del significado lingüístico contextualizado y flexible, infraespecificado en las propias palabras y asignado únicamente en contexto ${ }^{53}$.

Los significados 'permanentes' de la lengua se construyen repetidamente en situaciones comunicativas de interacción, social y culturalmente situadas ${ }^{54}$. La sociolingüística cognitiva propone, en este sentido, incluir variables contextuales, sociales y regionales ${ }^{55}$, para poder discernir empíricamente qué parte de la variación observada se debe a cuestiones conceptuales y qué parte a motivos externos; hecho que, como se podrá deducir, tiene una relevancia máxima.

El amplio componente metodológico de los estudios sociolingüístico-cognitivos demuestra un defensa explícita del análisis empírico, mayoritariamente cuantitativo. En un modelo teórico como este en el que todos los conocimientos del ser humano afectan al uso de la lengua ${ }^{56}$, son necesarios métodos que incluyan todos los factores internos y externos al fenómeno estudiado, y que desentrañen eficazmente qué parte de la variación responde a cada uno. Para ello, se utilizan métodos de análisis multivariante, que permiten medir simultáneamente el peso de diversos factores.

A diferencia de la sociolingüística tradicional, que suele basarse en entrevistas o conversaciones grabadas, la sociolingüística cognitiva utiliza técnicas de extracción masiva de datos no elicitados, propias de la lingüística de corpus. Habitualmente, no incluyen trabajo de campo, ni recogida directa de datos (con excepciones) ${ }^{57}$, aunque la diversidad de corpus es, cada vez más, uno de sus objetivos metodológicos, puesto que la información derivada de cada uno es limitada ${ }^{58}$.

\subsection{Líneas de investigación en sociolingüística cognitiva}

Geeraerts y Kristiansen ${ }^{59}$ destacan tres líneas principales de desarrollo de la sociolingüística cognitiva: 1) la que plantea modelos teóricos sobre el papel de los factores sociales en el desarrollo de la lengua ${ }^{60} ; 2$ ) la que investiga la variación de

\footnotetext{
${ }^{52}$ E. Rosch (1978); B. Berlin y P. Kay (1969); D. Geeraerts, S. Grondelaers, y P. Bakema (1994).

${ }^{53}$ K. Janicki (2006).

${ }^{54}$ C. Sinha (2007); J. Zlatev (2005); E. Itkonen (2003); P. Harder (2003).

${ }^{55}$ G. Kristiansen y R. Dirven (2008).

${ }^{56}$ D. Glynn (2010), p. 6.

${ }^{57}$ L. Impe, D. Geeraerts, y D. Speelman (2009); G. Kristiansen (2003); J. Robinson (2010).

${ }^{58}$ K. Heylen, J. Tummers, y D. Geeraerts (2008).

${ }^{59}$ D. Geeraerts y G. Kristiansen (2014), pp. 7-9.

${ }^{60}$ C. Sinha (2007); J. Zlatev (2005); E. Itkonen (2003); P. Harder (2003); etc.
} 
fenómenos de significado en relación con variables externas ${ }^{61} \mathrm{y}$, en particular, la relación entre la variación formal y la semántica ${ }^{62} ; \mathrm{y}$, finalmente, 3) la línea dirigida a estudiar cómo los hablantes perciben y categorizan la variación ${ }^{63}$.

La segunda línea incluye los estudios de variación léxico-semántica, objeto de este artículo. En el plano semasiológico, un objetivo básico es determinar el peso específico de los distintos significados de una categoría léxica (o "prominencia semasiológica"). Así, Robinson ${ }^{64}$ analiza el adjetivo awesome en un corpus de entrevistas y demuestra la importancia de la edad en la preferencia por términos referidos a significados más centrales (great), o más periféricos (terrible), que puede indicar un cambio en curso en la estructura semasiológica del adjetivo. Por su parte, Zhang et al. ${ }^{65}$ investigan qué factores influyen en el uso de nombres propios de capital de país como metonimia del concepto de 'gobierno', en un corpus de periódicos de China Continental y de Taiwán. Sus resultados apuntan a razones ideológicas y políticas.

En el plano onomasiológico, predominan los estudios de variación formal dedicados a medir la convergencia o divergencia de variedades geográficas de una misma lengua ${ }^{66}$. Un ejemplo excepcional de variación onomasiológica conceptual, el nivel menos atendido, es el estudio de Grondelaers y Geeraerts ${ }^{67}$ sobre la variación de 'cáncer' en un corpus escrito, para determinar si los niveles de especificidad en la expresión (específico: breast cancer; genérico: cancer; vago: disease) se ven influidos por el tema del artículo y su perspectiva más o menos impersonal. Los resultados muestran una tendencia a la hiperonimia en contextos en que se relata una experiencia personal, atribuida a un efecto del tabú sobre el nivel intermedio.

Estos estudios explican detalladamente su metodología de selección y codificación de ocurrencias; paso sin duda necesario para garantizar que el proceso de clasificación de variables sea transparente, coherente y comprensible. Este es el requerimiento propio del estudio de los niveles léxico y semántico que tienen, por fin, abierto el camino en sociolingüística. Los trabajos dentro de este paradigma se multiplican, demostrando su utilidad para el estudio de estas variables en perspectiva social, especialmente en relación con las categorías macrosociológicas.

${ }^{61}$ J. Robinson (2010); A. Soares da Silva (2005); E. Zenner, D. Speelman, y D. Geeraerts (2012); W. Zhang, D. Speelman, y D. Geeraerts (2011); etc.

62 D. Geeraerts, S. Grondelaers, y P. Bakema (1994); K. Heylen, Y. Peirsman, y D. Geeraerts (2008); T. Ruette, D. Speelman, y D. Geeraerts (2011); D. Speelman, S. Grondelaers, y D. Geeraerts (2003); etc.

${ }^{63}$ D. Geeraerts (2003); G. Kristiansen (2003, 2010); etc.

64 J. Robinson (2010).

${ }^{65} \mathrm{~W}$. Zhang et al (2011).

${ }^{66}$ D. Geeraerts, S. Grondelaers, y D. Speelman (1999); A. Soares da Silva (2010).

${ }^{67}$ S. Grondelaers y D. Geeraerts (1998). 
En la introducción, mencionaba que el estudio de la variación semántica simboliza un cambio de etapa en sociolingüística, junto con las variables microsociológicas, propias de los estudios de variación conocidos como "la Tercera Ola" ${ }^{68}$. Estos estudios están orientados a comprender qué consiguen los hablantes, como agentes sociales, con la variación en sus discursos. Esta perspectiva apenas está incorporada a la sociolingüística cognitiva, que explica la variación con patrones macrosociológicos (nación, edad, etc.). En las páginas siguientes expondré el argumento de Eckert, con la intención de incorporar sus reflexiones, finalmente, a la propuesta de este trabajo.

\section{La Tercera Ola y el significado social de la variación observada.}

Aunque la sociolingüística no tiene una teoría del significado lingüístico, la disciplina sitúa en primer plano la teoría del significado social. A pesar de su diversidad, las subdisciplinas sociolingüísticas mantienen el interés original por el estudio de las correlaciones entre lengua y sociedad ${ }^{69}$, ya que al sociolingüista le interesa el ser humano como animal que habla, piensa, se comunica y vive en sociedad $^{70}$.

Para la sociolingüística, la variación lingüística no es libre, o fruto del azar, sino que refleja, reproduce y construye significado social. Esto se traduce, cuantitativamente, en una relación entre la frecuencia de aparición de determinadas variantes y factores externos ${ }^{71}$ como el origen geográfico, la edad, la clase social, el sexo/género, la etnia, etc., determinantes en la construcción de la identidad ${ }^{72}$ y que afectan al uso de la lengua. Labov ${ }^{73}$ incluyó además la variable "estilo", relacionada con el uso de variedades más o menos formales por una misma persona, según la atención prestada al habla en diferentes situaciones comunicativas. El papel que ocupan hoy estos factores en la teoría ha evolucionado. Esta evolución se puede dividir en Tres Olas, no estrictamente cronológicas, de los estudios de variación ${ }^{74}$, según se interprete el significado de la variación: como un reflejo de categorías macrosociológicas (Primera Ola); como índice de categorías locales (Segunda Ola); como práctica social, indicadora y reproductora, indirectamente, de ciertas categorías (Tercera Ola).

La Primera Ola se inicia con el estudio variacionista de la ciudad de Nueva York $^{75}$. En los inicios, la motivación era estudiar las relaciones directas entre el uso

${ }^{68}$ P. Eckert (2005; 2009).

${ }^{69}$ F. Coulmas (1998), ver introducción.

${ }^{70}$ Como expresa P. Trudgill en su introducción a U. Ammon et al. (2004), p. 5.

${ }^{71}$ W. Labov (1966a).

72 J. M. Hernández Campoy (2011); R. B. Le Page y A. Tabouret-Keller (1985).

${ }^{73}$ W. Labov (1966b).

${ }^{74}$ P. Eckert (2005; 2009).

${ }^{75}$ W. Labov (1966b). 
lingüístico y las variables macrosociológicas (edad, sexo, clase social, etnia), entendidas como grandes bloques estructuradores de la sociedad. Esta etapa inicial fue criticada por su incapacidad para explicar, exclusivamente a través de patrones macrosociológicos, qué hacían los hablantes con las distintas variables lingüísticas en distintos puntos de la estructura ${ }^{76}$. Como respuesta, surgió la Segunda Ola, con enfoque etnográfico e interesada por la variación a nivel local. Consideran la existencia de categorías locales entre las variables macrosociológicas y el hablante, definidas ad hoc para cada grupo estudiado. En su investigación del instituto de Belten High, Eckert ${ }^{77}$ trabaja con las categorías locales Jocks y Burnouts, que remiten a los adolescentes de clase media y trabajadora, respectivamente. Otros ejemplos representativos de esta ola son los estudios de redes sociales en Belfast de Lesley Milroy ${ }^{78}$, las observaciones de Trudgill ${ }^{79}$ acerca del prestigio encubierto de las variantes vernáculas en hombres de clase media en Norwich o, en nuestro país, los trabajos de Villena Ponsoda ${ }^{80}$. La sociolingüística variacionista hispánica se ha movido hasta ahora mayoritariamente entre estas dos olas, como se puede observar en obras colectivas, manuales o estados de la cuestión que recogen los avances de la disciplina ${ }^{81}$.

La Tercera Ola avanza hacia una visión más flexible de la variación lingüística, no tan centrada en la estructura social. El cambio fundamental está en la relevancia que adquiere el concepto de 'práctica estilística' ${ }^{82}$, y en la inclusión de la agentividad de los hablantes dentro de la estructura social. Eckert ${ }^{83}$ subraya la repercusión de "la teoría de la práctica" de Pierre Bourdieu ${ }^{84}$ y la noción de habitus como centro de los procesos de reproducción social, situando la evolución de la sociolingüística dentro de un cambio de rumbo general de las ciencias sociales.

Los estudios de la Tercera Ola no niegan la importancia de la estructura social, sino que explican cómo aquella limita las posibilidades de la práctica y cómo la práctica produce y reproduce la estructura ${ }^{85}$. Se busca desentrañar qué significan los

\footnotetext{
${ }^{76}$ P. Brown y S. Levinson (1979).

${ }^{77}$ P. Eckert (1989; 2000).

${ }^{78}$ L. Milroy (1980).

${ }^{79}$ P. Trudgill (1972).

${ }^{80}$ J. A. Villena Ponsoda (2007).

${ }^{81}$ Entre otros, Blas Arroyo (2005); Cestero Mancera et al. (2006); J. A. Samper Padilla (2004).

${ }^{82}$ La 'práctica estilística' se refiere a las actividades, dinámicas y constantes, de creación de estilo por parte de los hablantes (aunque se relaciona también con prácticas extralingüísticas), vinculadas con significados sociales preexistentes, según Eckert (2005), p. 19.

${ }^{83}$ P. Eckert (2009),p. 14.

${ }^{84}$ P. Bourdieu (1972).

${ }^{85}$ P. Eckert (2009), p. 14.
} 
usos lingüísticos para las prácticas estilísticas en interacción, más que para las categorías, aunque sean locales ${ }^{86}{ }^{87}$. El uso de la lengua tiene un carácter performativo y, por tanto, la variación no es un mero reflejo de la estructura social, sino un recurso para poner en práctica determinadas acciones, cualidades o posiciones, que conforman un entramado de significados microsociológicos. Para entender cómo se vinculan los significados sociales locales con los macrosociológicos, se introduce el concepto de 'indexicalidad indirecta ${ }^{98}$, principio según el cual las formas lingüísticas no remiten a las categorías sociales directamente, sino indirectamente, a través de otras acciones realizadas discursivamente, como la toma de 'posiciones'.

La 'posición' (en inglés, stance) ${ }^{89}$ es un mecanismo mediador entre la actuación individual y el significado social. Aunque hay interpretaciones más estrictas ${ }^{90}$, $\mathrm{Jaffe}^{91}$ propone que las posiciones que un hablante construye interaccionalmente en el discurso pueden ser muy variadas: de 'experto', de 'élite' ${ }^{92}$, etc. Según Kiesling ${ }^{93}$, la posición sería la indexicalidad de primer orden, es decir, la que motiva la variación en primer lugar. Las posiciones que un hablante toma habitualmente configuran su "estilo personal", socialmente motivado, y podrían convertirse a su vez en índice de identidades distintas (o 'personas' o 'tipos sociales'), por la práctica y la repetición ${ }^{94}$.

En suma, se explica el significado de la variación de forma más matizada: las estrategias lingüísticas de los hablantes contribuyen a la toma de posiciones que, por la práctica, remiten a tipos sociales (locales), asociados con determinadas categorías macrosociológicas (ver Figura 1). Por ejemplo, Qing Zhang ${ }^{95}$ estudia cómo los yuppies de Pekín usan las vocales finales y la $/ \mathrm{z} /$ interdental (características típicamente pekinesas, relacionadas en particular con dos tipos locales, smooth operator y alley saunterer, y ciertas cualidades urbanas) para crear un estilo nuevo, que les sirve de demarcación social como élite económica.

${ }^{86}$ P. Eckert (2009), p. 15.

${ }^{87}$ Según la autora, la raíz de esta evolución teórica estaba de hecho en el primer estudio de W. Labov (1963) en Martha's Vineyard, que sienta las bases de lo que serán, años después, los aportes de la Segunda y la Tercera Olas.

${ }^{88}$ E. Ochs (1992); M. Silverstein (1992; 2003).

${ }^{89}$ Mi traducción.

${ }^{90}$ J. W. Du Bois (2007).

${ }^{91}$ A. Jaffe (2009).

92 ibíd., 8-9.

93 S. Kiesling (2009), p. 172.

${ }^{94}$ E. Ochs (1992).

${ }^{95}$ Q. Zhang (2005). 
Figura 1 Niveles de significado de la variación lingüística (interpretación según los estudios de variación de la Tercera Ola).

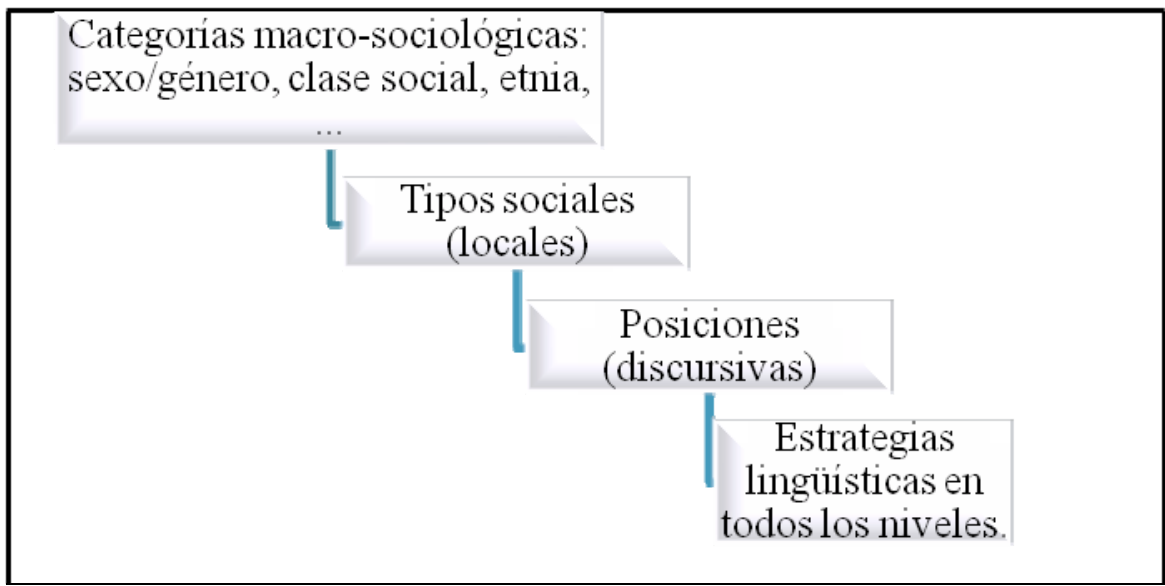

La variación lingüística se entiende como un sistema indexical cargado de significados, que inserta ideología en el lenguaje, permitiendo a su vez que los actos lingüísticos actúen sobre la sociedad ${ }^{96}$. La base de las prácticas estilísticas está en las oposiciones ideológicas que se dan en las interacciones con otros hablantes ${ }^{97}$, ya que estos despliegan sus propios estilos, provocando una sucesión de ajustes en la conversación. En estas situaciones de encuentro es donde se manifiestan las diferencias en los usos lingüísticos, a las que se les puede otorgar significado social.

La capacidad de reconocer características lingüísticas socialmente diversas hace que se puedan representar identidades distintas a la propia (por ejemplo, de otro dialecto), a través de rasgos estereotípicamente relacionados con ellas. En contra de la tradición variacionista, Eckert reclama que estos usos "a conciencia", al servicio de representaciones estereotipadas, son extremadamente reveladoras del orden social y, por tanto, son parte de la actividad estilística tanto como las actividades más automáticas.

Este giro hermenéutico, muy resumido, propone una visión menos esencialista y rígida de la sociolingüística, que diferencia a la Tercera Ola de la Segunda, pero sobre todo de la Primera. En este punto se entenderá por qué en sociolingüística cognitiva se echa de menos una mayor repercusión de estos avances interpretativos.

${ }^{96}$ P. Eckert (2009), p. 21.

${ }^{97}$ J. Irvine (2001). 
Las variables que se incluyen en la mayor parte de los estudios sociolingüísticocognitivos son macrosociológicas y su repercusión en la variación se interpreta como un reflejo del grupo al que se pertenece, como en la Primera Ola, sin cuestionamiento ${ }^{98}$. Una visión más coherente con la sociolingüística actual debería incluir un modelo basado en la práctica estilística e incorporar las teorías de la Tercera Ola en cuanto a la interpretación del significado social de la variación.

\section{La propuesta: una sociolingüística cognitiva de la Tercera Ola}

Para no "reinventar la rueda sociolingǘstica" 99 , cualquier estudio que adopte una perspectiva de variación debería incluir una reflexión profunda sobre qué significa esa variación. En esta línea, para responder a la pregunta de investigación inicial, “¿Qué puede ofrecer la sociolingüística a la sociolingüística cognitiva?”, considero que la sociolingüística puede ofrecer una reflexión teórica sobre la interpretación del significado de la variación, con implicaciones para la formulación del discurso teórico, para los objetivos de la disciplina y para el aparato metodológico diseñado para conseguirlos.

\subsection{Discurso teórico}

En ocasiones, armonizar discursos teóricos interdisciplinares puede ser una tarea compleja, pero este no es el caso. El discurso de la sociolingüística cognitiva es, en realidad, más coherente con los estudios de la Tercera Ola que con una visión más estanca de la variación, puesto que se basa en la flexibilidad, la performatividad y el no esencialismo.

La Tercera Ola propone una visión del comportamiento lingüístico que encaja dentro de una teoría social no solo lingüística, ya que introduce el estudio de la lengua en una reflexión global sobre el individuo, donde todo acto lingüístico contribuye a la práctica estilística ${ }^{100}$. Se puede decir que aplica una teoría maximalista del significado social, así como la lingüística cognitiva propone una visión maximalista del significado lingüístico. Este aspecto establece el primer fundamento teórico para una sociolingüística cognitiva de la Tercera Ola: la lengua es principalmente semántica ${ }^{101}$ y siempre construye significado social ${ }^{102}$.

Además, en ambas destacan la subjetividad y la interpretación como elementos de la comunicación lingüística. Las prácticas estilísticas mencionadas se dan en interacción y desde una perspectiva, la del hablante, que, a través de todos los recursos lingüísticos, toma posiciones según el contexto ${ }^{103}$. Ello implica una

\footnotetext{
${ }^{98}$ S. Gries (2013), p. 6.

${ }^{99}$ P. Eckert (2009), p. 2.

100 ibíd.

${ }^{101}$ D. Geeraerts y G. Kristiansen (2014), p. 3.

102 P. Eckert (2009), p. 21.

103 ibíd., p. 22.
} 
interpretación de la situación por parte del hablante, coherente con la idea de "filtro de la realidad" que supone el proceso de conceptualización ${ }^{104}$. La inclusión del aparato cognitivo en el modelo sociolingǘstico tiene el potencial de eliminar el esquema determinista de los inicios, al contemplar un elemento mediador entre el hablante y los factores sociales, que les da un margen amplio de flexibilidad y variación individual, dentro de su contexto social y cultural. En este sentido, ambas teorías son pragmáticas y experienciales en esencia.

La naturaleza performativa de la lengua en uso es consustancial a la idea de la práctica estilística, puesto que el hablante hace cosas con palabras; concretamente, crea significado social en varios niveles de indexicalidad ${ }^{105}$. La performatividad subyace también a la noción de estructuración conceptual de la realidad ${ }^{106}$, propia de la lingüística cognitiva (y retomada en la sociolingüística cognitiva), puesto que en cada acto lingüístico el hablante construye significado lingüístico desde una perspectiva.

Finalmente, ambas teorías aplican el no esencialismo. Para ambas, el significado (lingüístico y social) está infraespecificado: para la lingüística cognitiva, en el nivel del significado semántico de los propios elementos lingüísticos ${ }^{107}$, ya que no es posible definirlos según características suficientes y necesarias. Por su parte, la Tercera Ola considera que el significado social de los elementos lingüísticos también está infraespecificado, por lo que su poder indexical no se puede definir $a$ priori $^{108}$. Las variantes no se asocian con determinadas categorías directamente, sino que adquieren significados concretos según la situación. En ambos casos, la potencialidad de los elementos lingǘsticos, tanto en su aspecto semántico como social, solo significa plenamente en contextos concretos.

\subsection{Objetivos comunes y específicos}

Según Eckert ${ }^{109}$, el objetivo de la Tercera Ola es demostrar, que la variación expresa la totalidad de las inquietudes sociales de una comunidad dada; que el uso de la variación no solo refleja, sino que construye significado social, y es una fuerza de cambio de la sociedad; $y$, finalmente, que el significado social de las variables individuales está infraespecificado y solo se completa en el contexto del discurso, y de forma crucial, en la construcción de los estilos de habla.

Por su parte, el programa sociolingüístico-cognitivo está orientado a salvar la distancia existente entre la sociolingüística y la lingüística cognitiva; al estudio de la lengua en contexto; $y$, finalmente, al desarrollo de métodos empíricos

\footnotetext{
104 D. Geeraerts y G. Kristiansen (2014).

105 P. Eckert (2009), pp.19-20.

106 D. Geeraerts y G. Kristiansen (2014).

${ }^{107}$ K. Janicki (2006), p. 18.

${ }^{108}$ P. Eckert (2009), p. 22.

${ }^{109}$ P. Eckert (2009), p. 21.
} 
multivariantes que permitan distinguir los distintos tipos de variación cognitiva y social ${ }^{110}$.

Además de los anteriores, un objetivo específico de la sociolingüística cognitiva de la Tercera Ola, sería demostrar, por primera vez, el poder indexical de la variación en el nivel semántico y su rol en las prácticas estilísticas. Algunos trabajos anteriores permiten probar esta hipótesis: en estudios sobre la variación de los conceptos sexuales, las variables microsociológicas demostraron ser explicativas de la variación semántica. Por ejemplo, la variable 'aborto' demostró estar asociada con la construcción discursiva de las posiciones pro- y antiabortistas de los hablantes, en interacción oral y escrita ${ }^{111}$.

\subsection{Implicaciones metodológicas}

\subsubsection{La variable sociolingüística}

La sociolingüística de la Tercera Ola de Eckert propone cambiar el foco de estudio $^{112}$, ya que cuestiona la idea variacionista de la equivalencia entre las variantes fonéticas, supuestamente carentes de significado lingüístico. Retomando la idea del simbolismo fonético ${ }^{113}$, Eckert ${ }^{114}$ demuestra que los hablantes construyen significados afectivos y estilísticos a partir de diferencias en variantes fonéticas, como la realización oclusiva de la /t/ intervocálica en inglés ${ }^{115} \mathrm{o}$ la variación en la cantidad de $/ \mathrm{o} / \mathrm{y} / \mathrm{ay} /{ }^{116}$.

Las consecuencias teóricas son cruciales: si las variantes de una variable fonética pueden expresar distintos significados lingüísticos (como el significado + afectivo, mencionado anteriormente), se incorpora un componente semántico en este nivel de variación, situándola en el mismo plano de dificultad que Beatriz Lavandera ${ }^{117}$ enunció para los fenómenos morfosintácticos, léxicos y semánticos. En ese caso, es necesario asumir que la equivalencia de significado lingüístico no es una aspiración realista, ni debe ser un criterio para seleccionar variables de estudio.

La Tercera Ola propone que el criterio de selección sea el 'significado potencial' de la variable (en el sentido de la capacidad de significar social y estilísticamente), más que cuestiones estructurales o de cambio lingüístico ${ }^{118}$. Aunque esto abre la puerta a variables nuevas, la selección dependerá obviamente de qué se entienda por 'significado potencial'. En el plano onomasiológico, por

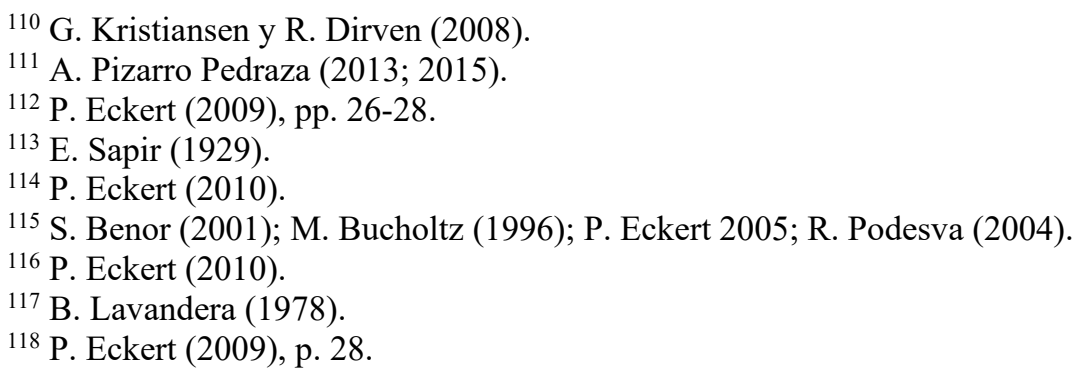


ejemplo, se puede entender el potencial de las variables según su variabilidad y su prominencia cultural como conceptos. En cuanto a la variabilidad, cuanto más heterogénea sea la expresión de un concepto, más posibilidades tendrá el análisis de ser rico en información sobre la categorización. Además, esta variabilidad puede estar relacionada con la existencia de distintas conceptualizaciones motivadas por factores culturales, como para los conceptos de 'gobierno' o 'mujer' ${ }^{119}$, o los nombres de 'religión' ${ }^{120}$ que han demostrado tener significados culturales $\mathrm{o}$ sociales. Otras áreas de la realidad que pueden tener significado potencial serían los modos de vida, las ideologías políticas, las emociones, las relaciones personales, la sexualidad, la muerte, etc.

Como reclamó García Mouton ${ }^{121}$ para la Dialectología, la colaboración interdisciplinar contribuye a una comprensión más profunda del fenómeno lingüístico. Si, para el estudio de la variación dialectal, "[e]xisten partes del vocabulario donde se perciben restos del trasfondo cultural de creencias antiguas y en las que la incidencia del folklore es más clara que en otras" ${ }^{122}$, parece razonable esperar que los conceptos relevantes para el análisis sociolingüístico (generalmente urbano) remitan a parcelas de la realidad prominentes en las comunidades de estudio. La sensibilidad ante estas categorías es crucial para seleccionar las que impliquen mayor significado potencial. En definitiva, la opción de una variable u otra deberá basarse en un conocimiento no únicamente lingüístico, sino cultural, social, histórico, etc., que permita localizar las áreas de interés en una comunidad dada.

Yendo más allá, la elección del objeto de estudio debería hacerse, en la medida de lo posible, desde una perspectiva crítica y ética; en línea con el compromiso con el ser humano y su contexto social habitual en sociolingüística ("principio de la deuda contraída" de Labov ${ }^{123}$; o "principio de gratuidad" de Wolfram ${ }^{124}$ ). La utilidad del estudio sociolingüístico no es solo lingüística, por lo que no es una cuestión menor dedicar el esfuerzo a áreas relevantes para la comunidad estudiada.

\subsubsection{Recogida de datos}

Como ya he mencionado, la metodología de recogida de datos mayoritaria de la sociolingüística cognitiva es la utilización de corpus disponibles. Puesto que la recogida de este tipo de datos tiene virtudes evidentes, quisiera centrar la atención sobre métodos más costosos en recursos, pero que presentan otro tipo de ventajas.

\footnotetext{
119 W. Zhang (2013).

${ }^{120}$ Y. Peirsman, K. Heylen, y D. Geeraerts (2010).

${ }^{121}$ P. García Mouton (1987a).

122 P. García Mouton (1987b), p. 189.

${ }^{123}$ W. Labov (1982), p. 173.

${ }^{124}$ W. Wolfram (1993), p. 227.
} 
La creación de corpus ad hoc presenta problemas difícilmente eludibles, de entre los que destaca la inversión de tiempo, tanto en el trabajo de campo como en la recogida de datos (a partir de entrevista u otros métodos) y en el procesamiento (normalmente, mediante transcripción) ${ }^{125}$. Además, estos corpus suelen ser más reducidos, y la cantidad de datos obtenida menor, especialmente para fenómenos poco recurrentes, como los de variación semántica. No obstante, una planificación basada en hipótesis sociolingüísticas convenientemente planteadas puede reducir este problema y permite de hecho recoger variantes semánticas en uso de forma controlada $^{126}$. Algunas metodologías pueden ser muy sofisticadas y sus resultados provechosos, como la de entrevista ${ }^{127}$.

Estas limitaciones se compensan por la riqueza de cierta información social, difícil de conseguir con corpus en línea, y que constituye la ventaja principal de la recogida de datos propios. Estos métodos permiten controlar ciertas condiciones, aumentando notablemente las posibilidades de obtener información social de los informantes, y no dejándola al azar, según la disponibilidad de los corpus en línea, muchas veces anónimos. En todo caso, es necesaria una reflexión crítica sobre los datos utilizados, puesto que de ellos depende el análisis y, por tanto, el alcance de las conclusiones de cada investigación.

\subsubsection{Consecuencias para el análisis y la interpretación}

El análisis de la sociolingüística cognitiva de la Tercera Ola debe poder responder a los objetivos de investigación planteados. En concreto, el estudio de los dos elementos que hemos considerado más relevantes de la nueva sociolingüística, la variación semántica y las variables microsociológicas, implica una combinación de mecanismos cualitativos y cuantitativos. La metodología mixta es necesaria para responder a los objetivos de análisis y comprender los significados microsociológicos.

La metodología cualitativa se aplica en dos fases del análisis. Para ciertos fenómenos de variación semántica, la detección y clasificación de variantes es un proceso manual difícilmente reemplazable por técnicas automáticas, especialmente en el caso de la onomasiología conceptual, extremadamente variable. Además, las variables microsociológicas, no pueden ser obtenidas automáticamente. Clasificar las posiciones que toman los hablantes conlleva un análisis cualitativo de sus discursos. En la fase de 'operacionalización', las clasificaciones citadas, semánticas y sociales, se traducen a variables operativas que puedan cuantificarse.

La cuantificación es la base de la metodología sociolingüístico-cognitiva, ya que investiga la prominencia de las variantes en términos de frecuencias. Para la

\footnotetext{
125 S. A. Tagliamonte (2006).

126 A. Pizarro Pedraza (2013).

${ }^{127}$ C. Briggs (1986); K. Hazen (2000); F. Moreno Fernández (2011); S. A. Tagliamonte (2006); también contestada, W. Labov (2001); L. Milroy (1978); N. Wolfson (1976).
} 
onomasiología conceptual, ausente hasta ahora en sociolingüística, Grondelaers y Geeraerts ${ }^{128}$ señalan tres factores que intervienen simultáneamente en la elección de una expresión concreta para determinado referente y que se determinan por su frecuencia: primero, la prominencia semasiológica del referente con respecto a la estructura semasiológica de la categoría; segundo, la prominencia onomasiológica de la categoría léxica nombrada por la expresión concreta; y tercero, los factores externos.

Los métodos multivariantes, como la regresión logística, permiten medir el peso específico de cada factor (interno o externo) sobre las preferencias semánticas ${ }^{129}$. Además de contemplar las posibles interacciones entre las variables externas para aproximarse al significado social de forma detallada, como en los modelos de efectos fijos ${ }^{130}$, los modelos de efectos mixtos permiten incluir variables aleatorias, como los informantes, para analizar la variación a nivel individual ${ }^{131}$.

Aunque el uso de métodos estadísticos requiera la operacionalización de realidades que no son numéricas, la cuantificación es un medio, pero no un fin en sí mismo: la interpretación del significado de la variación empieza tras la obtención de resultados.

El estudio de la variación debe ir encaminado a comprender su significado, en términos de su poder indexical y de su contribución a las prácticas sociales. Especialmente en el nivel semántico, si partimos de que la conceptualización está socialmente motivada, nada impide pensar que la variación semántica también les sirve a los hablantes para construir sus identidades en el discurso ${ }^{132}$.

En este sentido, la sociolingüística cognitiva de la Tercera Ola debe suavizar el papel de los factores macrosociológicos, e interpretar la variación en términos más locales, atendiendo a las posiciones y a las prácticas estilísticas. Prioritariamente, debe examinar el terreno aún poco transitado de la variación semántica y su uso en la práctica estilística, espacio virgen todavía por explorar.

\section{Conclusiones}

Señalaba al principio que nos encontramos en un momento más flexible y menos esencialista de la sociolingüística, relacionado con la posibilidad de estudio de la variación semántica, introducida por la sociolingüística cognitiva, y con la interpretación de la variación como práctica social, como en la Tercera Ola. Tras la explicación concisa de ambas corrientes, he propuesto un modelo teórico 'más sociolingüístico' de sociolingüística cognitiva, combinándolo con los presupuestos de la Tercera Ola, para conjugar el gran avance que suponen ambas para la

\footnotetext{
${ }^{128}$ S. Grondelaers y D. Geeraerts (2003), p. 72.

${ }^{129}$ D. Glynn (2010), p. 6.

${ }^{130}$ H. Baayen (2008), p. 214.

${ }^{131}$ Ibíd. p. 263; S. A.Tagliamonte y H. Baayen (2012); S. Gries (2013).

${ }^{132}$ Como en A. Pizarro Pedraza (2013, 2015).
} 
disciplina. Ello responde a una voluntad de flexibilizar la interpretación sociolingüística, que refleje la libertad con la que los hablantes construyen sus identidades discursivamente. Además de los objetivos de ambos modelos, la sociolingüística cognitiva de la Tercera Ola tiene el objetivo específico de estudiar el poder indexical de la variación semántica, aún por investigar.

El modelo tiene características teóricas como el maximalismo, el subjetivismo o la performatividad; y también metodológicas: la selección de la variable por su significado potencial, interpretada aquí en términos de relevancia cultural y social; la recogida de datos mediante métodos de campo; las metodologías de análisis mixto orientadas a un estudio multivariante que incluya variables microsociológicas, interacciones y variables aleatorias; y finalmente, la interpretación de la variación en términos de sus significados locales y cotidianos.

Esta propuesta, como he procurado defender, permitirá comprender con mayor flexibilidad y matices las opciones lingüísticas (por fin, en todos los niveles) de los hablantes que, al ser entendidos como agentes sociales, serán tratados con mayor justicia, al reconocérseles su capacidad de ser creativos. Las compatibilidades teóricas y las posibilidades prácticas permiten afirmar que una sociolingüística cognitiva de la Tercera Ola es un programa coherente y realizable, con la potencialidad de explicar los hechos lingüísticos desde una perspectiva más completa, que sitúe la práctica estilística en el centro del círculo que forman cognición y sociedad ${ }^{133}$.

\section{Obras citadas}

ALLWOOD, Jens: «Meaning potential and context. Some consequences for the analysis of variation in meaning», en Cognitive approaches to lexical semantics, editado por Hubert Cuyckens, René Dirven, y John R Taylor, Berlín: Mouton de Gruyter. 2009.

AMMON, Ulrich, Norbert DitTMAR, Klaus J MATTHEIER, y Peter TRUDGILl, eds.: Sociolinguistics. Soziolinguistik. An international handbook of the science of language and society. Ein Internationales Handbuch zur Wissenschaft von Sprache und Gesellschaft, Berlín, Walter de Gruyter, 2004.

BAAYEN, Harold: Analysing linguistic data. A practical introduction to statistics, Cambridge, Cambridge University Press, 2008.

BENOR, Sarah: «Sounding learned: The gendered use of $/ \mathrm{t} /$ in Orthodox Jewish English», en Penn working papers in linguistics: selected papers from NWAV

\footnotetext{
${ }^{133}$ La primera versión de este trabajo se realizó con el apoyo de una beca predoctoral JAE en el Instituto de Lengua, Literatura y Antropología (ILLC) del Consejo Superior de Investigaciones Científicas (CSIC). La presente versión se ha finalizado con el apoyo de una beca postdoctoral del programa Move-In Louvain. Les agradezco a mis directores de tesis, Pilar García Mouton y Eugenio Bustos Gisbert, a los miembros de mi tribunal de tesis y a los dos revisores anónimos sus comentarios para mejorar este artículo.
} 
2000, Filadelfia, Universidad de Pensilvania, 2001.

Berlin, Brent, Dennis H Breedlove, y Peter H RAVEN: «General principles of classification and nomenclature in folk biology», American Anthropologist 75 (1), (1973), pp. 214-42.

BERLIN, Brent, y Paul KAY: Basic color terms. Their universality and evolution, Berkeley/Los Ángeles, University of California Press, 1969.

BORREGO NIETO, Julio: «Dificultades en el estudio sociolingüístico del léxico», en II Encuentro de lingüistas y filólogos de España y México, editado por Alonso et al., Salamanca, Universidad de Salamanca y Junta de Castilla y León, 1994, pp. 119-31.

Blas Arroyo, José Luis: Sociolingüistica del español. Desarrollos y perspectivas en el estudio de la lengua española en contexto social, Madrid, Cátedra, 2005.

BourdiEU, Pierre: Esquisse d'une théorie de la pratique, París, Éditions du Seuil, 1972.

BOYE, Kasper y Peter HARDER: «Complement-taking predicates: usage and linguistic structure», Studies in Language 31 (3), (2007), pp. 569-606.

Briggs, Charles L.: Learning How to Ask: A Sociolinguistic Appraisal of the Role of the Interview in Social Science Research, Cambridge, Cambridge University Press, 1986.

BROwn, Penelope, y Stephen C. LEVINSON: «Social structure, groups and interaction», en Social markers in speech, editado por H. Giles y K. R. Scherer, Cambridge, Cambridge University Press, 1986.

BUCHOLTZ, Mary: «Geek the girl: language, femininity and female nerds», en Gender and belief systems. Proceedings of the Fourth Berkeley women and language conference, editado por N. Warner, J. Ahlers, L. Bilmes, M. Oliver, S. Wertheim, y M. Chen, Berkeley, Berkeley Women and Language Group, 1996, pp. 119-31.

Cestero, Ana $\mathrm{M}^{\mathrm{a}}$, Isabel Molina, y Florentino PARedes, eds.: Estudios sociolingüísticos del español de España y de América, Madrid, Arco Libros, 2006.

COUlmas, Florian, ed.: The Handbook of Sociolinguistics, Oxford (RU) /Cambridge (MA), Wiley-Blackwell, 1998.

CUENCA, Maria Josep, y Joseph HILFERTY: Introducción a la lingüística cognitiva, Madrid, Ariel Letras, 2013.

CUYCKEnS, Hubert, René DIRVEN, y John R TAYLOR, eds.: Cognitive approaches to Lexical Semantics, Berlín, Mouton De Gruyter, 2009.

Du BoIS, John W.: «The Stance Triangle», en Stancetaking in Discourse, editado por Robert Englebretson, Ámsterdam/Filadelfia, John Benjamins, 2007.

ECKERT, Penelope: Jocks and Burnouts, Social categories and identity in the high school, Nueva York, Teachers College Press, 1989.

— : Linguistic variation as social practice, Oxford, Blackwell, 2000.

: «Variation, convention, and social meaning», Annual meeting of the 
Linguistic Society of America, Oakland, CA, 2005.

- : «Three waves of variation studies», http.//www.stanford.edu/ eckert/PDF/ThreeWavesofVariation.pdf. 2009.

- : «Affect, Sound symbolism, and variation», University of Pennsylvania Working Papers in Linguistics 15 (2), (2010), Article 9.

ESCORIZA MORERA, Luis: «La variación de expresión en el plano léxico, dificultades y perspectivas», Lingüística 28 (1), (2012), pp. 247-73.

ETXEBARRIA AROSTEgUI, Maitena: «Disponibilidad léxica en escolares del país vasco, variación sociolingüística y modelos de enseñanza bilingüe», Revista Española de Lingüistica 26 (2), (1996), pp. 301-25.

FILLMORE, Charles J.: «Frame Semantics», en Linguistics in the morning calm, editado por Linguistic Society of Korea, Seoul, Hanshin, 1982, pp. 111-38.

GARCÍA MoUTON, Pilar: «Dialectología y cultura popular. Estado de la cuestión», Revista de dialectología y tradiciones populares, 49, (1987a), pp- 49-71.

: «Motivación en los nombres de los animales», Lingüistica Español Actual 9 (9), (1987b), pp. 189-97.

GEERAERTS, Dirk: «Lectal variation and empirical data in cognitive linguistics», en Cognitive Linguistics. Internal dynamics and interdisciplinary interactions, editado por Francisco Ruiz de Mendoza Ibáñez y Sandra Peña Cervel, Berlín/Nueva York, Mouton de Gruyter, 2005, pp. 163-89.

— : «Introduction», en Cognitive Linguistics. Basic readings, editado por Dirk Geeraerts, Vol. 34, Berlín, Mouton De Gruyter, 2006.

-: «Schmidt Redux, how systematic is the linguistic system if variation is rampant?» en Language usage and language structure, editado por Kasper Boye y Elisabeth Engberg-Pedersen, Berlín/Nueva York, De Gruyter Mouton, 2010, pp. 237-62.

GEERAERTS, Dirk, Stefan GRONDELAERS, y Peter BAKEMA: The structure of lexical variation, Berlín, Mouton De Gruyter, 1994.

GeEraerts, Dirk, Stefan Grondelaers, y Dirk SpeElman: Convergentie en divergentie in de nederlandse woordenschat, een onderzoek naar kleding-en voetbalternem, Ámsterdam, Meertens Instituut, 1999.

GEERAERTS, Dirk, y Gitte KRISTIANSEN: "Cognitive linguistics and language variation», en Bloomsbury Companion to Cognitive Linguistics, editado por Jeanette Littlemore y John Taylor, Londres, Bloomsbury, 2014, pp. 202-217.

GeEraerts, Dirk, Gitte Kristiansen, y Yves Peirsman, eds. Advances in Cognitive Sociolinguistics, Berlín/Nueva York, De Gruyter Mouton, 2010.

GLYNN, Dylan: «Corpus-driven cognitive semantics. Introduction to the field», en Quantitative cognitive semantics. Corpus-driven approaches, editado por Dylan Glynn y Kerstin Fischer, Berlín/Nueva York, Mouton De Gruyter, 2010.

GRIES, Stefan: «The sources of variability relevant to the (cognitive) sociolinguist, and quantitative corpus methods to handle them», Journal of Pragmatics 52 (6), (2013), pp. 5-16. 
GRONDELAERS, Stefan, y Dirk GEERAERTS: «Vagueness as a euphemistic strategy», en Speaking of emotions, conceptualisation and expression, editado por Angeliki Athanasiadou y Elżbieta Tabakowska, Berlín/Nueva York, Mouton de Gruyter, 1998, pp. 357-74.

: «Towards a pragmatic model of cognitive onomasiology», en Cognitive approaches to Lexical Semantics, editado por Hubert Cuyckens, René Dirven, y John R Taylor, Berlín, Mouton de Gruyter, 2003, pp. 67-92.

HALLIDAY, M. A. K.: «On grammar and grammatics», en Functional descriptions, Theory in Practice, editado por Ruqaiya Hasan, Carmel Cloran, y David G Butt, Ámsterdam/ Filadelfia, John Benjamins, 1996.

HARDER, Peter: «The status of linguistic facts, rethinking the relation between cognition, social institution and utterance from a functional point of view», Mind and Language 18, (2003), pp. 52-76.

HASAN, Ruqaiya: Semantic variation, meaning in society and in sociolinguistics, Londres, Equinox Publishing Ltd., 2009.

HAZEN, Kirk: «The role of researcher identity in conducting sociolinguistic research, a reflective case study», Southern Journal of Linguistics 24, (2000), pp. 10320.

HERNÁNDEZ CAMPOY, Juan Manuel: «Variation and identity in Spain», en The Handbook of Spanish Sociolinguistics, editado por Manuel Díaz-Campos, Londres, Blackwell Publishing, 2011.

HERNÁNDEZ MUÑOZ, Natividad: Hacia una teoría cognitiva integrada de la disponibilidad léxica: el léxico disponible de los estudiantes castellanomanchegos, Salamanca, Universidad de Salamanca, 2006.

Heylen, Kris, Yves Peirsman, y Dirk GeERAerts: «Automatic Synonymy Extraction», en Computational Linguistics in the Netherlands 2007, editado por Suzan Verberne, Hans van Halteren, y Peter-Arno Coppen, Ámsterdam, Rodopi, 2008, pp. 101-16.

HeYlen, Kris, José Tummers, y Dirk GeERAERTs: «Methodological issues in corpus-based cognitive linguistics», en Cognitive Sociolinguistics, language variation, cultural models, social systems, editado por Gitte Kristiansen y René Dirven, Berlín/Nueva York, Mouton de Gruyter, 2008, pp. 91-128.

HollmanN, Willem B.: «Constructions in Cognitive Sociolinguistics», en The Oxford handbook of construction grammar, editado por T. Hoffmann y G. Trousdale, Oxford, Oxford University Press, 2013, pp. 491-509.

IBARRETXE-ANTUÑANO, Iraide, y Javier VALENZUELA: Lingüistica cognitiva, Barcelona, Anthropos, 2012a.

: «Lingüística cognitiva: origen, principios y tendencias», en Lingüistica cognitiva, editado por Iraide Ibarretxe-Antuñano y Javier Valenzuela, Barcelona, Anthropos, 2012b, pp. 1-16.

IMPE, Leen, Dirk GEERAERTS, y Dirk SPEELMAN: «Mutual intelligibility of standard and regional Dutch language varieties», International journal of 
humanities and arts computing 2, (2009), pp. 101-17.

IRVINE, Judith T.: «Style as distinctiveness, the culture and ideology of linguistic differentiation», en Stylistic variation in language, editado por Penelope Eckert and John Rickford, Cambridge, Cambridge University Press, 2001.

ITKONEN, Esa: What is language? A study in the Philosophy of linguistics, Turku, Åbo Akademis tryckeri, 2003.

JAFFE, Alexandra: Stance. Sociolinguistic Perspectives, Nueva York, Oxford University Press, 2009.

JANICKI, Karol: Language misconceived. Arguing for applied cognitive sociolinguistics, Mahwah, New Jersey, Lawrence Erlbaum Associates, 2006.

KIESLING, Scott: «Style as stance. Stance as the explanation for patterns of sociolinguistic variation», en Stance. Sociolinguistic Perspectives, editado por Alexandra Jaffe, Nueva York, Oxford University Press, 2009.

KRISTIANSEN, Gitte: «How to do things with allophones, linguistic stereotypes as cognitive reference points in social cognition», en Cognitive models in language and thought, ideologies, metaphors, and meanings, editado por René Dirven, Roslyn Frank, y Martin Pütz, Berlín/Nueva York, Mouton de Gruyter, 2003.

: «Towards a usage-based cognitive phonology», International journal of English studies 6 (2), (2006), pp. 107-40.

Kristiansen, Gitte, y René DIRVEN: Cognitive Sociolinguistics. Language variation, cultural models, social systems, Berlín, De Gruyter Mouton, 2008.

LABOV, William: «The social motivation of a sound change», Word, no. 19, (1963), pp. 273-309.

- : "The linguistic variable as a structural unit», Washington Linguistics Review 3, (1966a), pp. 4-22.

- The social stratification of English in New York City, Washington, D.C., Center for Applied Linguistics, 1966b.

: Sociolinguistic patterns, Filadelfia, University of Pennsylvania Press, 1972a.

-: Some principles of linguistic methodology», Language in Society 1 (1), (1972b), pp. 97-120.

: "Objectivity and commitment in linguistic science, The case of the Black English trial in Ann Arbor», Language in Society, no. 11, (1982), pp. 165-202. -: "The anatomy of style-shifting», en Style and sociolinguistic variation, editado por Penelope Eckert y John R. Rickford, Cambridge y Nueva York, Cambridge University Press, 2001.

Labov, William, Sharon Ash, Maya Ravindranath, Tracey Weldon, Maciej BARANOWSKID, y Naomi NAGYE: «Properties of the sociolinguistic monitor», Journal of Sociolinguistics 15 (4), (2011), pp. 431-63.

Lavandera, BEATRIZ R.: «Where does the sociolinguistic variable stop?» Language in Society 7 (02), (1978), pp. 171-82. 
Le Page, R B, y A Tabouret-Keller: Acts of identity, Cambridge, Cambridge University Press, 1985.

LÓPEZ MORALES, Humberto: «El estudio de la variación léxica», en Estudios sociolingüísticos del español de España y de América, editado por Ana María Cestero Mancera, Isabel Molina Martos, y Florentino Paredes García, Madrid, Arco Libros, 2006.

MiLROY, Lesley: «Belfast, change and variation in an urban vernacular», en Sociolinguistic patterns in British English, editado por Peter Trudgill, University Park Press, 1978.

- Language and social networks, Londres/Baltimore, Basil Blackwell/University Park Press, 1980.

Moreno FERnÁNDEZ, Francisco: Principios de sociolingüística y sociología del lenguaje, $4^{\mathrm{a}}$ edición, Barcelona, Ariel, 2009.

- Sociolingüistica cognitiva. Proposiciones, escolios y debates, Madrid/Frankfurt, Iberoamericana / Vervuert, 2012.

OCHS, Elinor: «Indexing Gender», en Sex and Gender Hierarchies, editado por B Miller, Cambridge, Cambridge University Press, 1992.

AiJón OlIVA, Miguel Ángel, y María José SERRANO: «Las bases cognitivas del estilo lingüístico», Sociolinguistic Studies 4 (1), (2010), pp. 115-44.

Peirsman, Yves, Kris HeYlen, y Dirk Geeraerts: «Applying Word Space Models to Sociolinguistics. Religion Names before and after 9/11», en Advances in Cognitive Sociolinguistics, editado por Dirk Geeraerts, Gitte Kristiansen, e Yves Peirsman, Berlín/Nueva York, De Gruyter Mouton, 2010, pp. 111-37.

PIZARRo PedrazA, Andrea: Tabú y eufemismo en la ciudad de madrid. Estudio sociolingüístico-cognitivo de los conceptos sexuales, Universidad Complutense, 2013.

- : "Who said 'abortion'? Semantic variation and ideology in Spanish newspapers' online discussions», Australian Journal of Linguistics 35 (1), (2015), pp. 53-75.

PODESVA, Robert: «On constructing social meaning with stop release bursts», Sociolinguistics Symposium 15, Newcastle upon Tyne, 2004.

PÜTZ, Martin, Justyna ROBINSON, y Monika REIF: «The emergence of cognitive sociolinguistics. an introduction», Special Issue of Review of Cognitive Linguistics 10 (2), (2012), pp. 241-63.

RoBINSON, Justyna: «Awesome Insights into Semantic Variation», en Advances in Cognitive Sociolinguistics, editado por Dirk Geeraerts, Gitte Kristiansen, y Yves Peirsman, Berlín/Nueva York, Mouton de Gruyter, 2010.

RODRÍGUEZ GONZÁLEZ, Félix, y Bernard L. ROCHET: «Variación sociolingüística en el léxico, mujer, esposa y señora en el español contemporáneo», Analecta Malacitana, Revista de la sección de filología de la facultad de filosofia y letras 22 (1), (1999), pp. 159-78. 
Rosch, Eleanor: «Principles of Categorization», en Cognition and Categorization, editado por Eleanor Rosch y B. Lloyd, Hillsdale, New Jersey, Laurence Erlbaum Associates, 1978.

Ruette, Tom, Dirk SPeElman, y Dirk Geeraerts: «Measuring the Lexical Distance between Registers in National Varieties of Dutch», en Linguas Pluricêntricas. Variação Linguística E Dimensóes Sociocognitivas, editado por Augusto Soares da Silva, Amadeu Torres, y Miguel Gonçalves, Braga, Publicações da Faculdade de Filosofia, Universidade Católica Portuguesa, 2011, pp. 541-54.

SAMPER PADILlA, José Antonio: «Disponibilidad léxica y sociolingüística», en Discurso y sociedad, contribuciones al estudio de la lengua en contexto social, editado por José Luis Blas Arroyo, Manuela Casanova Ávalos, y Mónica Velando Casanova, 2006.

-: «La sociolingüística en España», Español Actual, Revista de español vivo, no. 98, (2012), pp. 39-70.

SANKOFF, David, Pierrette ThIBAUlt, y Hélène BÉRUBÉ: «Semantic field variability», en Linguistic variation, models and methods, editado por David Sankoff, Nueva York, Academic Press, 1978, pp. 23-43.

SAPIR, Edward: «A study in phonetic symbolism», Journal of experimental psychology 12, (1929), pp. 225-39.

- : «Language», en Selected writings of Edward Sapir in Language, culture and personality, editado por David G. Mandelbaum, Berkeley/Los Ángeles/Londres, University of California Press, 1949.

SHARIFIAN, Farzad: Cultural conceptualisations and language. Theoretical framework and applications, Ámsterdam/Filadelfia, John Benjamins, 2011.

Silva-Corvalán, Carmen: Sociolingüistica y pragmática del español, Washington D.C., Georgetown University Press, 2001.

SilversteIn, M.: «The uses and utility of ideology, some reflections», Pragmatics 2 (3), (1992), pp. 311-23.

- :Indexical order and the dialectics of sociolinguistic life», Language \& Communication, no. 23, (2003), pp. 193-229.

SINHA, Chris: «Cognitive linguistics, psychology and cognitive science», en The Oxford Handbook of Cognitive Linguistics, editado por Dirk Geeraerts y Hubert Cuyckens, Nueva York, Oxford University Press, 2007.

SOARES DA SILVA, Augusto: «Para o estudo das relações lexicais entre o português europeu e o português do Brasil, elementos de sociolexicologia cognitiva e quantitativa do português», en Actas Do XX Encontro Nacional Da Associação Portuguesa de Linguística, editado por Inês Duarte y Isabel Leiria, Lisboa, Associação Portuguesa de Lingüística, 2005.

- : «Lexical convergence and divergence in Portuguese», en Advances in Cognitive Sociolinguistics, editado por Dirk Geeraerts, Gitte Kristiansen e Yves Peirsman, Berlín/Nueva York, Mouton de Gruyter, 2010. 
SpeElman, Dirk, Stefan Grondelaers, y Dirk GeERAERTS: «Profile-based linguistic uniformity as a generic method for comparing language varieties», Computers and the Humanities 37, (2003), pp. 317-37.

TAGLIAMONTE, Sali A.: Analysing sociolinguistic variation, Cambridge, Cambridge University Press, 2006.

TAgLiAmOnTE, Sali A, y Harold BAAYEN: «Models, forest and trees of york english. was/were variation as a case of study for statistical practice», Language Variation and Change 24 (2), (2012), pp. 135-78.

TAYLOR, John R.: Linguistic categorization, prototypes in linguistic theory, Oxford, Clarendon Press, 1989.

TRUDGILL, Peter: «Sex, covert prestige and linguistic change in the urban British English of Norwich», Language in Society 1 (2), (1972), pp. 179-95.

VILLENA PONSODA, Juan Andrés: «Redes sociales y variación lingüística: el giro interpretativo en el variacionismo sociolingüístico», Actas del VI Congreso de Lingüistica General, Santiago de Compostela, 3-7 de mayo de 2004, coord. por Pablo Cano López, 3, 2007, pp. 2769-2806.

WEINER, E. Judith, y William LABOV: «Constraints on the agentless passive», Journal of Linguistics 19 (01), (1983), pp. 29-58.

WEINREICH, Uriel, William LABOV, y Marvin HERZOG: «Empirical foundations for a theory of language change», en Directions for Historical Linguistics, A Symposium, editado por Winfred P. Lehmann e Yakov Malkiel, Austin, University of Texas Press, 1968.

WOLFRAM, Walt: «Ethical Considerations in language awareness programs», Issues in Applied Linguistics, no. 4, (1993), pp. 225-55.

WOLFSON, Nessa: «Speech events and natural speech, some implications for sociolinguistic methodology», Language in Society 5 (2), (1976), pp.189-209.

ZENNER, Eline, Dirk SpEelman, y Dirk GeERAERTS: «Cognitive sociolinguistics meets loanword research, measuring variation in the success of anglicisms in dutch», Cognitive Linguistics 23, (2012), pp. 749-92.

- : «A sociolinguistic analysis of borrowing in weak contact situations, english loanwords and phrases in expressive utterances in a Dutch reality TV show», International Journal of Bilingualism, 19(3), (2014), pp. 333-346.

ZHANG, Qing: «A Chinese yuppie in Beijing, phonological variation and the construction of a new professional identity», Language in Society 34, (2005), pp. 431-66.

ZHANG, Weiwei, Dirk SPEELMAN, y Dirk GeERAERTS: «Variation in the (non)metonymic capital names in mainland Chinese and Taiwan Chinese», Metaphor and the Social World 1, (2011), pp. 90-112.

ZLATEV, Jordan: «What's in a schema? Bodily mimesis and the grounding of language», en from perception to meaning, image schemas in cognitive linguistics, editado por Beate Hampe, Berlín/Nueva York, Mouton de Gruyter, 2005. 